\title{
Le pharmacien, éducateur des patients
}

\author{
par Lalitha Raman-Wilms
}

$\mathrm{D}$ ans ce numéro du $J C P H$, la pharmacienne, Marie Craig, nous fait part de son expérience en éducation et en soutien des patients à la clinique de l'arthrite de Newmarket, en Ontario $^{1}$. Ce rôle très important pour les pharmaciens est clairement reconnu par les associations professionnelles et universitaires canadiennes, de même qu'à l'échelle mondiale. En 1997, l'Organisation mondiale de la Santé (OMS) présentait le rôle d'éducateur comme l'une des sept responsabilités clés des pharmaciens ${ }^{2,3}$. Dans le cadre de leurs fonctions, les pharmaciens continuent de donner aux patients et à leurs soignants de l'information et des conseils sur les médicaments. Malgré cela, la non-observance thérapeutique reste élevée, certaines études signalant que plus de $50 \%$ des patients ne prennent pas leurs médicaments tels que prescrits ${ }^{4}$. Outre ses conséquences négatives sur la qualité de vie des patients, la non-observance thérapeutique a aussi des répercussions économiques évaluées à 100 milliards de dollars par année aux États-Unis 5 .

Alors pourquoi la non-observance thérapeutique est-elle toujours aussi fréquente? Les raisons sont complexes. Malgré que les consommateurs aient accès à une foule de renseignements sur les médicaments et sur les maladies imprimés et en ligne - , certaines fois l'information dont ils ont besoin n'est pas complète ou peut même être contradictoire, selon les sources. Dans de tel cas, les patients s'en remettent à eux-mêmes pour juger si un médicament pourra ou non leur être utile, ce qui se traduit par l'autogestion de la prise de médicaments, quelques fois à l'insu des professionnels de la santé qui les soignent ${ }^{6,7}$.

Plusieurs facteurs expliquent pourquoi un patient n'est pas fidèle à son traitement. Tout comme nous saisissons l'importance de comprendre comment le patient se sent face à sa maladie (sa réponse subjective face à la maladie), nous devons aussi comprendre comment il se sent face aux médicaments qu'il doit prendre. Tout cela est conditionné par ses valeurs culturelles et ses croyances face à la santé qu'il a acquises tout au long de sa vie, de même que ses expériences passées avec les professionnels de la santé et les médicaments. ont une influence sur l'observance du patient au traitement.

Des recherches montrent que les patients doivent pouvoir s'expliquer de façon logique pourquoi ils doivent prendre leurs médicaments, ce qui peut avoir une influence sur le moment auquel ils les prendront, voire même sur leur décision de les prendre ou non ${ }^{6}$. Afin d'avoir une incidence positive sur l'expérience pharmacothérapeutique des patients, les pharmaciens et les autres professionnels de la santé ne doivent plus simplement se contenter de donner de l'information générale sur les médicaments et s'attendre à ce que les patients suivent leur traitement, mais ils doivent plutôt " apprendre comment ils peuvent participer au processus de prise de décision du patient en matière de médicaments $"{ }^{9}$. Cela nécessite de personnaliser l'éducation, comme le fait Marie Craig avec ses patients atteints d'arthrite. L'OMS utilise le terme " éducation thérapeutique des patients " pour décrire cette activité, la définissant comme une éducation visant à transmettre au patient les compétences et les stratégies dont il a besoin pour gérer et adapter au mieux sa propre maladie chronique ${ }^{10}$.

Dans la foulée de cette approche, le Groupement Pharmaceutique de l'Union Européenne a émis récemment une déclaration sur le rôle du pharmacien dans l'éducation thérapeutique des patients ${ }^{11}$. Cette déclaration énonce que les patients doivent être formés à acquérir les compétences dont ils ont besoin pour gérer eux-mêmes leur maladie et adapter leur traitement à leur situation personnelle. En outre, les professionnels de la santé doivent être formés à concevoir des outils d'éducation efficaces pour les patients ${ }^{11}$.

La recherche a permis jusqu'à ce jour d'apprécier toute la complexité du processus de prise de décision du patient et d'expliquer pourquoi l'information à elle seule peut être insuffisante pour la plupart des patients. Des études plus poussées sur le sujet permettront aux pharmaciens de mieux comprendre comment traduire de façon optimale l'information sur les maladies et sur les médicaments en information que les patients pourront comprendre et mettre à profit. Il ne fait cependant nul doute que l'éducation personnalisée des patients, comme le fait Marie Craig, peut être très efficace pour les aider à " acquérir [...] une compétence d'autonomie $»^{11}$, et leur permettre de prendre des décisions pharmacothérapeutiques éclairées, en collaboration avec leurs professionnels de la santé.

[Traduction par l'éditeur] 


\section{Références}

1. Practice spotlight: Marie Craig and The Arthritis Program (TAP) at Southlake Regional Health Centre. Can J Hosp Pharm 2009;62(2):148-149.

2. The role of the pharmacist in the health care system. Preparing the future pharmacist: curricular development. Report of a Third WHO Consultative Group on the Role of the Pharmacist, Vancouver, Canada, du 27 au 29 aout 1997. Rapport WHO/PHARM/97/599. Organisation mondiale de la Santé; 1997. Publié à www.who.int/medicinedocs/fr/d/Js2214e/. Consulté le 17 février 2009.

3. Un nouvel outil pour renforcer le rôle du pharmacien dans les soins de santé. Genève (Suisse) : Organisation mondiale de la Santé; 23 novembre 2006. Publié à www.who.int/mediacentre/news/new/2006/nw05/ fr/index.html. Consulté le 11 janvier 2009.

4. Dunbar-Jacob J, Dwyer EJ. Compliance with antihypertensive regimens: a review of the research in the 1980s. Ann Behav Med 1991;13(1):32-39.

5. Donovan JL, Blake DR. Patient non-compliance: deviance or reasoned decision-making? Soc Sci Med 1992;34(5):507-513.

6. Bajcar J. Task analysis of patients' medication-taking practice and the role of making sense: a grounded theory study. Res Social Adm Pharm 2006;2(1):59-82.

7. Bikowski RM, Ripsin CM, Lorraine VL. Physician-patient congruence regarding medication regimens. J Am Geriatr Soc 2001;49(10):1353-1357.

8. Understanding the patient's medication experience. Dans : Cipolle RJ, Strand LM, Morley PC, éditeurs. Pharmaceutical care practice - the clinician's guide. 2 éd. New York (NY): McGraw-Hill Companies, Inc; 2004. p 102-117.

9. Vermeire E, Hearnshaw H, Van Royen P, Denekens J. Patient adherence to treatment: three decades of research. A comprehensive review. J Clin Pharm Ther 2001;26(5):331-342.
10. Organisation mondiale de la Santé. Therapeutic patient education. Continuing education programmes for health care providers in the field of prevention of chronic diseases. Report of a WHO working group. Copenhague (Danemark): Bureau régional de l'Europe; 1998. Publié à http://www.euro.who.int/ document/e63674.pdf. Consulté le 11 janvier 2009.

11. Déclaration du GPUE : Le rôle du pharmacien dans l'éducation thérapeutique des patients. Réf. : 08.11.18F 008. Bruxelles (Belge) : Groupement Pharmaceutique de l'Union Européenne; novembre 2008. Publié à www.gpue.eu/Portals/8/documents/2008/Position\%20Papers/ 08.11.18F\%20008\%20PGEU\%20Statement\%20on\%20Therapeutic\% 20Education_FINAL.pdf. Consulté le 11 janvier 2009.

Lalitha Raman-Wilms, B. Sc. (Phm.), Pharm. D., FCSHP est professeure agrégée et directrice, Division de la pratique pharmaceutique, Faculté de pharmacie Leslie Dan de l'Université de Toronto à Toronto, en Ontario. Elle est également rédactrice adjointe du $\mathrm{JCPH}$.

Adresse de correspondance :

Docteure Lalitha Raman-Wilms

Leslie Dan Faculty of Pharmacy

University of Toronto

144 College St.

Toronto (ON)

M5S 3M2

Courriel : I.raman.wilms.a@utoronto.ca 